\title{
Knowledge of iron-containing foods among pregnant women in Lagos, Nigeria
}

\section{Abstract \\ Introduction}

Anaemia in pregnancy is a global public health problem and about half of all anaemia cases among pregnant women in developing countries are attributable to iron (Fe) deficiency. Perhaps, nutritional knowledge on the vitality of Fe-containing foods among pregnant women is lacking. In the light of the perpetually high burden of anaemia in pregnancy in Nigeria, we conducted a study to assess the knowledge of Fe-containing foods among pregnant women in Lagos, Nigeria.

\section{Materials and Methods}

Four hundred and thirty two (432) pregnant women in the 2nd and 3rd trimester of pregnancy (across eight Primary Health Centers in a local government area) from Lagos Nigeria identified through multi-stage sampling method completed validated semi-structured questionnaires on socio-demographic characteristic and knowledge of Fe-containing foods using a validated 10-question knowledge scale (designed by nutrition experts) measured on a 2-point scale. Knowledge score (KS) was categories as poor (2 test was used to evaluate the association between age groups, education, marital status and knowledge of Fe-containing foods (at $\mathrm{P}<0.05)$.

\section{Results}

Mean age of all respondents was $28.5 \pm 4.6$ years and $43.3 \%$ were below 28 years. Mean gestational age was $31.3 \pm 4.1$ weeks, $6.5 \%$ and $1.9 \%$ reported having a primary formal education only and never married respectively. Mean knowledge score of all respondents was $6.7 \pm 2.2$ and $34.7 \%$ had poor $\mathrm{KS}$ about Fe-containing foods. While age of respondents was insignificantly associated with KS, proportion of pregnant women who had good KS was significantly higher $\left(\chi^{2}=6.6, \mathrm{P}=0.01\right)$ among those with at least a college education $(66.8 \%)$ compared to their counterpart having at a primary formal education only $(42.9 \%)$. In addition, proportion of respondents with poor KS was significantly lower $\left(\chi^{2}=9.9, \mathrm{P}=0.007\right)$ among those ever married $(34.8 \%)$ compared to respondents who never married $(75.0 \%)$.

\section{Conclusion}

Aside nutrition education, the vitality of adequate formal education for women prior to pregnancy and care giving roles cannot be undermined in advancing the knowledge of women on anemia and alleviating the burden anaemia in pregnancy in developing countries.

\section{Conflict of Interest}

There is no conflict of interest 